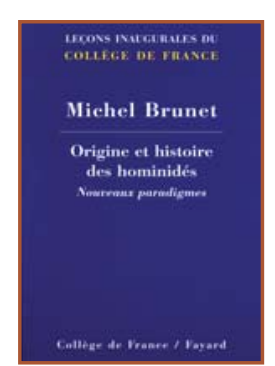

Origine et histoire des hominidés

Nouveaux paradigmes

Michel Brunet

Coll. Leçons inaugurales du Collège de France Paris, Collège de France/Fayard, 2008.

En 2002, Michel Brunet et son équipe mettaient au jour le plus ancien hominidé aujourd'hui connu : « Toumaï ». Cette découverte a bouleversé l'histoire de nos origines.

Jusqu'alors on situait l'apparition des premiers hommes en Afrique de l'Est, il y a trois ou quatre millions d'années, en relation avec un changement de climat: le remplacement de la forêt par la savane aurait favorisé la bipédie. Ce scénario

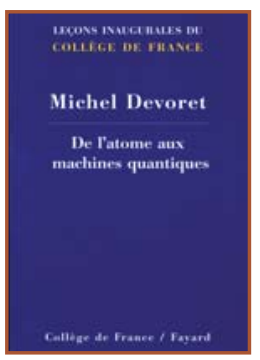

De l'atome aux machines quantiques

Michel Devoret

Coll. Leçons inaugurales du Collège de France

Paris, Collège de France/Fayard, 2008.

Par leur grand nombre d'atomes, les systèmes macroscopiques peuvent facilement être assemblés à partir de pièces indépendantes, tels les horloges et leurs rouages. Quant aux systèmes quantiques, ils présentent des pro-

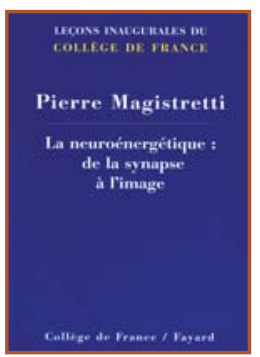

La neuroénergétique : de la synapse

à l'image

Pierre Magistretti

Coll. Leçons inaugurales du Collège de France

Paris, Collège de France/Fayard, 2008.

Comment les neurones reçoivent-ils l'énergie nécessaire à leur activité ? Mettant en évidence le rôle décisif des cellules gliales - jusqu'alors "parias - des études neurobiologiques -, les travaux de Pierre Magistretti et son équipe ont récemment permis de mieux comprendre ces

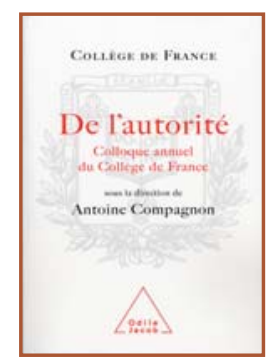

De l'autorité

Colloque annuel du Collège de France 2007 sous la direction du Pr Antoine Compagnon Paris, Éd. Odile Jacob, 2008.

De l'Antiquité au monde contemporain, dans toutes les cultures, l'autorité, c'est-à-dire la souveraineté, le sacré, le livre, le dogme, a fondé l'ordre social. Elle est donc partout et nulle part.

Le droit, la philosophie, la religion, la science politique, l'économie, la sociologie, sans omettre les sciences exactes : tous nos savoirs séduisant doit être abandonné : Toumaï a sept millions d'années, il vivait au Tchad, alors recouvert de lacs et de forêts, et il marchait sans doute sur ses deux pieds.

C'est l'histoire de cette découverte et de ce bouleversement de nos schémas théoriques que raconte ici Michel Brunet.

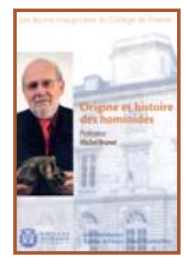

Un DVD comprenant le film de la leçon inaugurale, un entretien avec le Pr Yves Coppens, deux présentations, une par le Pr Armand de Ricqlès et une par le Pr Édouard Bard, est également disponible. Coproduction Collège de France-Cned - Doriane Films.

priétés qui les rendent naturellement réguliers et résistants au bruit, à la différence des systèmes classiques soumis au chaos. Les systèmes mésoscopiques sont à la fois macroscopiques par leur nombre d'atomes et quantiques par le comportement de leur degré de liberté : ils combinent les atouts de ces deux mondes.

processus neuroénergétiques : ils sont à la base des nouvelles techniques d'imagerie cérébrale fonctionnelle qui permettent de "voir » le cerveau fonctionner. Ils paraissent jouer un rôle décisif dans la maladie d'Alzheimer et la dépression, et leur lien avec l'inconscient freudien ouvre un champ de recherche inédit.

Pierre Magistretti retrace ici l'histoire mouvementée de ces découvertes, où l'ignorance et l'erreur ont parfois joué un rôle positif.

sont ici interrogés par les meilleurs spécialistes. Sont ainsi traitées des questions aussi diverses que l'autorité de la Constitution, ce qu'est une "haute autorité indépendante ", ce qu'était l'autorité du roi en Mésopotamie, le rôle de la tradition et l'origine du canon biblique, le statut de l'autorité dans les sciences, en médecine ou dans le domaine des climats par exemple, mais aussi la notion d'autorité morale, le "poids de l'autorité » dans la croyance, etc. 

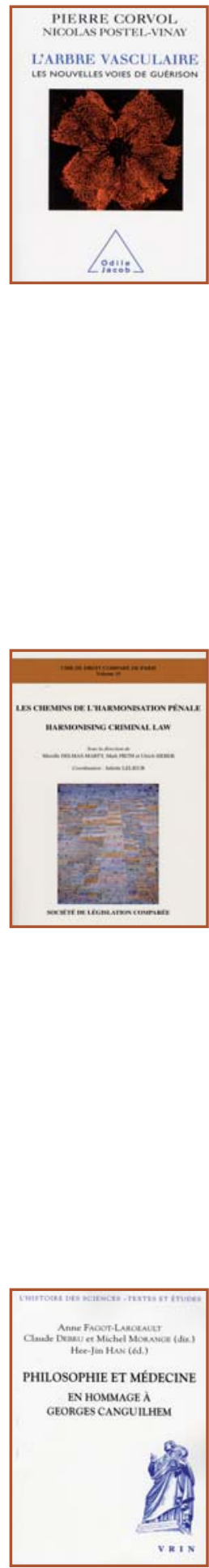

L'arbre vasculaire

Les nouvelles voies de guérison

Pierre Corvol, Nicolas Postel-Vinay

Paris, Éd. Odile Jacob, 2008.

En 1787, John Hunter utilisait pour la première fois le mot angiogenesis (angiogenèse) pour décrire les vaisseaux qui croissent dans les bois des cerfs. Deux siècles après, le premier facteur de croissance des vaisseaux sanguins est identifié. Depuis, la recherche sur les vaisseaux sanguins et lymphatiques a fait un bond en avant.

Nos idées sur l'arbre vasculaire se sont renouvelées. Dès la Renaissance, les grands anatomistes, Malpighi ou Vésale, ont représenté les vaisseaux à la manière d'un arbre. Naturalistes et physiologistes, dont Stephan Hales puis Claude Bernard, ont pris le chemin de l'expérimentation: mesurer la pression sanguine

Les chemins de l'harmonisation pénale Harmonising Criminal Law

Sous la direction de Mireille Delmas-Marty, Mark Pieth et Ulrich Sieber

Coordination : Juliette Lelieur

UMR de droit comparé de Paris, Volume 15 Paris, Société de législation comparée, 2008.

Alors que le droit de punir est traditionnellement considéré comme l'emblème de la souveraineté nationale, le droit pénal se trouve paradoxalement en première ligne de l'harmonisation du droit, au confluent de deux phénomènes apparemment convergents : l'universalisme juridique, qui fonde les droits de l'homme et la notion de crimes contre l'humanité, et la globalisation économique, qui facilite le développement d'une délinquance transnationale. À partir de champs d'expérimentation situés dans

\section{Philosophie et médecine}

en hommage à Georges Canguilhem

Anne Fagot-Largeault

Claude Debru et Michel Morange (dir.)

Hee-Jin Han (éd.)

Paris, Librairie philosophique J. Vrin, 2008.

Ouvrage publié avec le soutien du Collège de France

Une philosophie du vivant est affaire indécise, coincée entre chose et pensée, entre ceux qui optent pour le matérialisme et ceux qui perçoivent dans le vivant une âme ou un esprit, une réalité spirituelle. Conscient des enjeux, Canguilhem le résistant, le libertaire, trace son chemin en signifiant, au besoin avec une pointe d'agressivité, qu'il ne glissera pas plus sur une pente que sur l'autre. Il refonde la réflexion sur le vivant, d'une manière profondément originale, marquée par l'exigence de chez l'animal et la montée de la sève dans la tige des plantes. Les formes arborescentes qui ont intrigué et passionné ces chercheurs sont omniprésentes dans le monde animal, végétal et minéral.

Ces formes, ces canaux et ces vaisseaux, ces conduites de vie retrouvées à toutes les échelles de la nature servent une fonction commune : la communication. Relier l'oxygène ambiant au plus profond de nos cellules, les éléments nutritifs du sol aux feuilles de l'arbre, l'eau des rivières et des fleuves aux territoires qu'ils traversent.

S'intéresser aux vaisseaux, aux organes qu'ils irriguent, c'est découvrir les mécanismes ancestraux de leur formation qui ont précédé de loin l'émergence de l'homme sur terre. C'est aussi suivre une ère thérapeutique toute nouvelle avec ses répercussions sur une société prompte à récolter les fruits médicaux et financiers de l'arbre.

divers domaines (crimes contre l'humanité, traite des êtres humains, cybercriminalité, terrorisme, corruption, atteintes aux intérêts financiers de l'Union européenne, auxquels s'ajoutent des questions transversales comme les organisations criminelles ou les sanctions), ce livre présente des éléments pour une théorie de l'harmonisation pénale. Ces éléments sont présentés dans la perspective dynamique annoncée par la métaphore des chemins : d'abord les acteurs de la transformation (publics, civiques et économiques); puis les facteurs d'évolution; ensuite les processus d'interaction ; enfin les cinq modèles qui sous-tendent les pratiques pénales (souverainiste, universaliste, libéral, pluraliste à dominante légaliste et pluraliste à dominante humaniste). Les auteurs concluent l'ouvrage en esquissant une évaluation critique des pratiques pénales au regard de ces cinq modèles.

rigueur conceptuelle, par l'alliance de la philosophie et de l'histoire des sciences, et par une intelligence aiguë de l'acte médical.

Canguilhem, penseur aux intuitions abruptes, qu'il présentait avec une prudente modestie, avait à l'intérieur de la profession une présence forte, mais il n'était à aucun degré un philosophe médiatique ou mondain. Que reste-t-il de sa réflexion ? Comment sa pensée est-elle reçue par une nouvelle génération de philosophes qui ne l'ont pas connu personnellement, ni professionnellement ? C'est ce que ce recueil tente de préciser.

Ont contribué à ce volume: J.-G. Barbara, A. Benmakhlouf, J.-F. Braunstein, L. Cherlonneix, C. Debru, J.-C. Dupont, É. Giroux, H.-J. Han, N. Lechopier, A. Leplège, L. Montel, M. Morange, H. Schmidgen, F. Worms, In-Sok Yeo. 


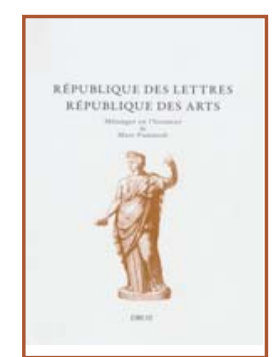

République des Lettres

République des Arts

Mélanges en l'honneur de Marc Fumaroli

Réunis et édités par Christian Mouchel et

Colette Nativel

Publié avec le soutien du Collège de France

Librairie Droz S.A., Genève, 2008.

Intitulé République des Lettres, République des Arts, ce volume de "Mélanges » témoigne du rayonnement scientifique de l'œuvre de Marc Fumaroli et manifeste les nombreux liens d'amitié qu'il a noués au long de sa carrière à la Sorbonne et au Collège de France, pendant ses séjours dans les universités d'Europe et d'Amérique, et qu'il continue d'entretenir depuis l'Académie française. Les trente-trois auteurs participant à cet ouvrage explorent les multiples aspects de l'histoire de la culture et des représentations, des formes symboliques et des genres d'expression qui ont suscité l'intérêt de Marc Fumaroli, de la Renaissance au XIXe siècle, en passant par l'âge Baroque et les Lumières. Institutions savantes et diffusion des doctrines ou des croyances; mythes littéraires et lieux communs de l'imagination profane ou sacrée ; figures du discours et catégories esthétiques; action et fonctions de la rhétorique ; rapports de la parole éloquente avec la raison politique et philosophique, avec la vie sociale et la création artistique : autant de sujets traversés par l'érudition et la pensée de Marc Fumaroli, dont les contributions ici réunies illustrent la fécondité.

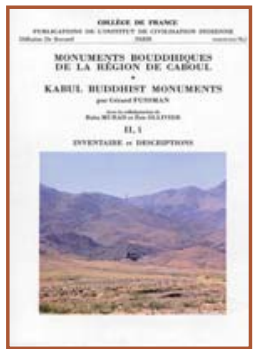

Monuments bouddhiques de la région de Caboul / Kabul Buddhist

Monuments II

Gérard Fussman avec la collaboration d'Éric Ollivier et de Baba Murad Publications de l'Institut de Civilisation Indienne du Collège de France, fasc. $76^{1}$ et 762, Paris, 2008.

Ce livre constitue la suite et le développement d'une étude parue en 1976 (Gérard Fussman et Marc Le Berre, Monuments bouddhiques de la région de Caboul, I, Le monastère de Gul Dara, Mémoires de la Délégation archéologique française en Afghanistan, tome XXII, Paris, Diffusion De Boccard), essentiellement consacrée à la publication de la fouille du monastère de Gul Dara. Y était annoncée une étude à venir sur les autres monuments bouddhiques de la région. On la trouvera dans cette seconde et dernière partie, inventaire critique de tous les monuments bouddhiques de la région de Caboul et du Koh Daman (région de Begram) connus à ce jour (2007). Ils sont localisés très précisément, décrits et replacés dans leur environnement géographique et historique. Une grande partie des données ici publiées étaient inédites. Une attention particulière a été portée à l'illustration - 97 planches en tout dont 16 en couleurs - que nous avons voulu aussi complète que possible. Tous les monuments ont été photographiés. Les mieux conservés ont fait l'objet de relevés architecturaux (plans, coupes, dessins du décor). Texte et illustrations ont été imprimés sur deux cahiers indépendants afin de faciliter l'indispensable aller-retour entre la description et l'image. Résumés anglais, persan et pashto.

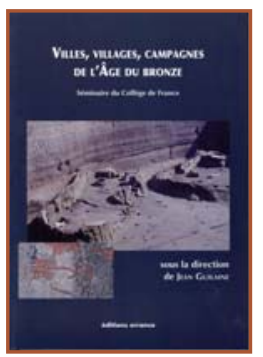

Villes, villages, campagnes de l'Âge du bronze

Séminaire du Collège de France

Sous la direction de Jean Guilaine

Paris, Éditions Errance, 2008.

Entre le monde urbanisé oriental et les communautés rurales d'Occident, entre "lettrés " et «barbares ", l'Âge du bronze est la période de tous les contrastes. Sur une vaste diagonale étirée du Pakistan à l'Atlantique, cet ouvrage aborde un ensemble de questions centrées autour de la notion d'espace, habité ou exploité : genèse des villes de l'Indus, relations agglomérations/ campagnes en Mésopotamie, développement et disparition des cités levantines, caractères de l'occupation du sol et de l'organisation de l'habitat de la Méditerranée centrale à l'Europe de l'Ouest, images de paysages ruraux et de leur parcellaire (Campanie, Normandie), embellie des Terramares, exploitation de la montagne (Alpes, Pyrénées), contraintes de l'environnement. Une gerbe d'exemples témoignant de la variété des choix culturels, des modèles d'habitat et d'aménagement des territoires. 


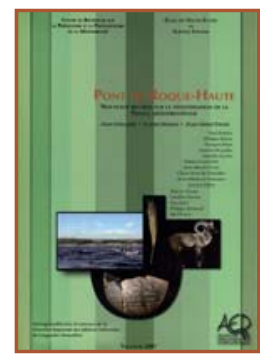

Pont de Roque-Haute

Nouveaux regards sur la néolithisation de la France méditerranéenne

Jean Guilaine, Claire Manen, Jean-Denis Vigne

Centre de Recherche sur la Préhistoire et la Protohistoire de la Méditerranée

École des Hautes Études en Sciences Sociales

Toulouse, Archives d'écologie préhistorique, 2007.

Cet ouvrage constitue la monographie du site de Pont de Roque-Haute (Portiragnes, Hérault), l'un des plus anciens établissements néolithiques du littoral méditerranéen français. Les vestiges mis au jour témoignent de l'installation, dans la première moitié du

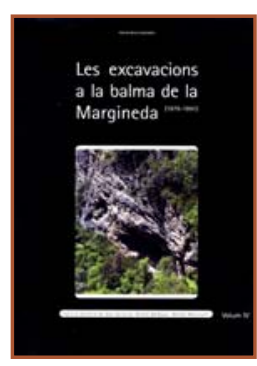

Les excavacions a la balma de la

Margineda (1979-1991)

Volum IV

Sota la direcció de Jean Guilaine, Michel

Barbaza i Michel Martzluff

Andorra, Edicions del Govern d'Andorra, 2007.

Avant-propos par Jean Guilaine, Michel Barbaza et Michel Martzluff

Ce quatrième volume, consacré aux recherches archéologiques poursuivies sur le site de la Balma de la Margineda, en Principauté d'Andorre, traite entièrement des couches profondes observées lors de nos interventions sur le terrain : les strates 7 à 12 . Dans ces dépôts inférieurs, seules les couches 7, 8,10 et, très partiellement, 9 peuvent être considérées comme anthropiques et recéler des vestiges, plus ou moins abondants, liés à des fréquentations et des activités humaines. Les couches 11 et 12 , seulement sondées, sont de ce point de vue stériles; leur présence n'est d'ailleurs que partielle car, sur une bonne partie de l'aire fouillée, la couche 10 repose sur une accumulation de gros blocs qui constitue l'assise de l'ensemble des strates archéologiques.

Les couches 7 à 10 ont la particularité de pouvoir être attribuées à une même entité 6e millénaire avant notre ère, d'un petit groupe humain en provenance probable de l'Italie centro-méridionale. Tout en conservant le mode de vie développé dans leur zone d'origine, ces pionniers néolithiques ont su s'adapter aux caractéristiques environnementales régionales et utiliser la diversité des ressources offertes. La vingtaine de contributions réunies propose une description de l'environnement dans lequel les Néolithiques ont développé leurs activités et une analyse de la façon dont ils ont exploité ce milieu, à partir des données matérielles et économiques. Dans la partie finale de synthèse, le site est replacé dans le scénario plus large de la néolithisation de la Méditerranée nord-occidentale.

culturelle: l'Azilien. Ainsi s'explique leur regroupement dans un même ouvrage. Ce découpage ne doit pas, toutefois, contribuer à exagérer les ruptures entre ces strates et celles décrites dans le tome précédent. Entre la couche 7 (traitée ici dans le contexte culturel de l'Azilien) et la couche 6 qui la surmonte et dont les données ont été livrées, dans le Tome III, avec les couches "mésolithiques ", il n'existe pas à notre avis de coupure brutale, mais bien un processus de continuité. Tout au long de l'Épipaléolithique de la Margineda, et en dépit de périodes temporaires de lacune, subsiste une longue tradition de fréquentation de l'abri, en rapport avec une économie d'exploitation de la montagne fondée sur la chasse et, plus que tout, sur la traque du bouquetin. Afin de maintenir une certaine unité dans la présentation des divers volumes, nous avons suivi, pour ces dépôts anciens, un ordre de présentation identique à celui adopté pour les strates supérieures, à savoir l'étude stratigraphique, l'approche géoarchéologique, l'énumération des datations isotopiques, la documentation archéologique, la cadre paléoenvironnemental, les vestiges végétaux et fauniques plus directement liés aux activités humaines.

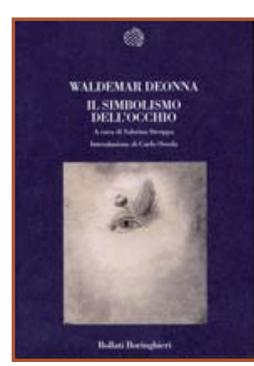

Il simbolismo dell'occhio

Waldemar Deonna

Texte établi par Sabrina Stroppa, Introduction de Carlo Ossola, Turin, Bollati Boringhieri, 2008, 346 p.

Opus vitae de Waldemar Deonna (1880-1959), archéologue, historien des arts 


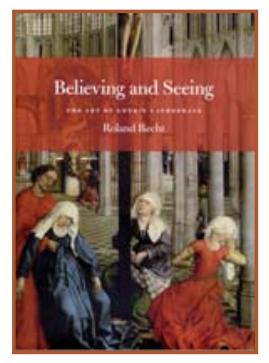

Believing and Seeing

The art of Gothic cathedrals

Roland Recht

Translated by Mary Whittall

Printed in the United States of America,

The University of Chicago Press, 2008.

Traduction anglaise de :

Le croire et le voir: L'art des cathédrales, XIIe-XVe siècle, Paris, Gallimard, 1999.

Les développements de la science médiévale et de la philosophie naturelle ont élevé la vue audessus des autres sens et ont fait d'elle le fondement de la vérité empirique. Dans le même temps, l'importance accordée par les chrétiens aux miracles, aux reliques et aux grands édifices a donné à ces principes une expression religieuse. Dans son étude pénétrante de l'art et de l'architecture gothiques, Roland Recht soutient que la place du voir comme clé de la connaissance religieuse a profondément affecté un vaste ensemble d'œuvres médiévales tardives.
Recht élargit les contours de l'étude du gothique et passe des grandes cathédrales françaises à d'autres édifices religieux importants d'Europe du Nord, d'Italie et d'Espagne. Il révèle comment leur architecture devient le support d'une profusion d'images qui rendent visibles les enseignements des écritures. L'orfèvrerie, par exemple, façonne des ostensoirs aux motifs élaborés et met en scène les reliques. Le progrès des techniques de production de verres teintés rejaillit sur l'art du vitrail. La sculpture gagne en expressivité et la peinture exploite toutes les ressources d'une palette enrichie pour rendre les sujets plus vivants. Recht pose sur ces œuvres un regard renouvelé. Il y voit un lien entre les pratiques dévotes de la fin du Moyen Âge et les théories de l'époque, qui donnaient au voir une place centrale. Il offre ainsi aux étudiants et aux chercheurs un outil nouveau et puissant pour aborder l'art et l'architecture gothiques.

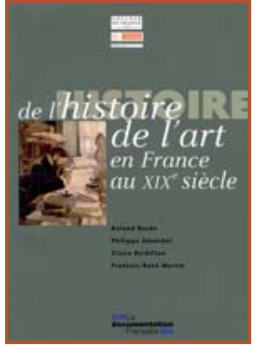

Histoire de l'histoire de l'art en France au XIXe siècle

Roland Recht, Philippe Sénéchal, Claire Barbillon, François-René Martin

Avec le concours du Collège de France, de la Fondation Hugot du Collège de France, de l'Institut national d'histoire de l'art et de l'École du Louvre

Paris, La Documentation Française, 2008.

L'histoire de l'histoire de l'art étudie toutes les formes de discours qui prennent pour objet les œuvres d'art, les conditions historiques et idéologiques dans lesquelles ces discours ont été élaborés, et les sujets de ces discours. Son développement depuis une quarantaine d'années est le résultat d'une crise profonde qui a affecté la discipline, tout comme l'ensemble des sciences humaines. Or une discipline qui perd ses certitudes s'interroge fatalement sur ses méthodes et plus encore sur sa propre histoire.

Ce qui est alors en jeu, ce sont les contours mêmes de l'histoire de l'art. Centrée avant tout sur l'établissement de catalogues et de biographies d'artistes, sur l'interprétation iconographique, sur l'étude matérielle des œuvres d'art, l'histoire de l'art est entrée dans les années 1970 dans une phase nouvelle. Des thèmes sont alors définis, qui avaient été jusque-là considérés comme devant rester à l'extérieur de son champ : par exemple, la théorie de la réception élaborée par la critique littéraire, qui fournit des outils remarquables à l'histoire de l'art ; ou l'« ère de la reproductibilité technique ", qui affecte jusqu'au statut même des œuvres reproduites ; ou encore, l'activité croissante des musées, qui contribue fortement à modifier le champ d'intervention où l'histoire de l'art s'est jusque-là cantonnée. L'intérêt porté aux grandes figures de la discipline, à l'idéologie qui sous-tend les discours sur l'art - que ce soient des livres ou des expositions, l'enseignement ou les autres formes de diffusion du savoir -, aux reproductions et au musée imaginaire, aux doctrines relatives au patrimoine monumental et muséal : tels sont quelques-uns des "genres » qui entraînent aujourd'hui l'histoire de l'art aux confins d'autres spécialités, déplacements ou élargissements dont elle tire le plus grand profit.

Le présent recueil, sous le titre Histoire de l'histoire de l'art en France au XIXe siècle, et qui réunit des contributions d'une trentaine d'historiens de l'art, quelques-uns venus de pays où l'historiographie de l'art est riche d'une longue tradition, voudrait contribuer à inscrire une réflexion sur ses méthodes et sur les figures qui les ont illustrées dans les priorités de cette toujours jeune science humaine qu'est l'histoire de l'art. 


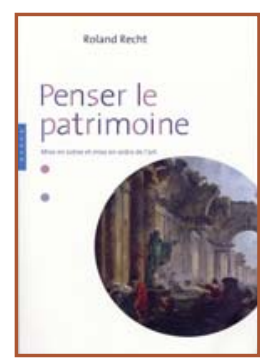

Penser le patrimoine

Mise en scène et mise en ordre de l'art

Roland Recht

Nouvelle édition enrichie

Paris, Éditions Hazan, 2008.

Dans la mesure où il procède à un transfert qui fait passer l'objet de sa destination cultuelle ou fonctionnelle primitive, au statut à la fois esthétique et historique d'œuvre d'art, le musée constitue une mise en scène et un dispositif parfaitement réglés. Le musée, le catalogue ou encore les ouvrages consacrés à l'art sont autant de mises en ordre discursif des œuvres d'art.

Dans une série d'études ponctuelles, Roland Recht analyse les changements qui affectent notre relation aux objets esthétiques, depuis l'Elysée d'Alexandre Lenoir jusqu'au Grand Louvre ouvert en 1993, dont la mise en scène déploie vers la ville un patrimoine-spectacle qui entre en contradiction avec la définition même du musée.

II s'interroge également sur la redoutable mutation qui gagne aujourd'hui quelques grandes institutions patrimoniales et sur les effets néfastes qu'elle exerce sur la vie de l'esprit.

L'auteur examine enfin quelques cas de "réhabilitations ", comme celle des primitifs ou de l'art roman, et montre ainsi comment l'histoire de l'art construit ses objets.

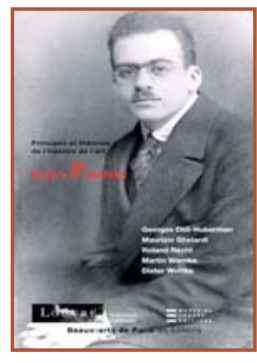

Relire Panofsky

Principe et théories de l'histoire de l'art Roland Recht, Martin Warnke, Georges

Didi-Huberman, Maurizio Ghelardi,

Dieter Wuttke

Conférences et colloques du Louvre

Paris, Ed Beaux-arts de Paris, 2008.

Professeur d'histoire de l'art à l'université de Hambourg de 1926 à 1934, Erwin Panofsky est étroitement lié à Ernst Cassirer et à Aby Warburg, mais il s'intéresse également aux courants formalistes issus de l'école de Vienne. Son nom est associé au décryptage de la représentation picturale au moyen de l'iconographie et à un important recours aux sources littéraires. II émigre aux États-Unis en 1934 et, à partir de là, il est considéré comme l'un des grands maîtres de l'histoire de l'art.

La postérité de sa pensée a suscité un questionnement sur ses fondements mêmes: existe-t-il une vérité historique en matière d'œuvres d'art et dans quelle mesure sommesnous capables de la saisir ?

Les communications réunies dans cet ouvrage, ainsi que la traduction pour la première fois en français de la postface à la réédition allemande de Hercule à la croisée des chemins, s'articulent autour de cette interrogation et invitent le lecteur à reconsidérer l'œuvre de Panofsky comme un apport capital à l'historiographie de l'art et des civilisations.

Enfin, un choix de lettres inédites offre un panorama succinct, mais capital pour comprendre les relations entre Panofsky et la France, sujet rarement abordé par la critique. Pour tous ceux qui s'intéressent à l'œuvre de Panofsky, ce recueil constitue désormais un outil indispensable.

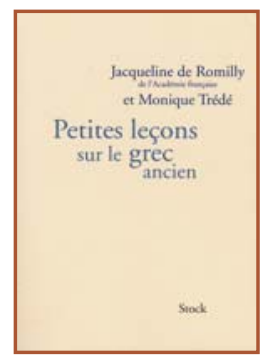

Petites leçons sur le grec ancien Jacqueline de Romilly, Monique Trédé Paris, Stock, 2008.

"Une langue, c'est beaucoup plus qu'une somme de règles grammaticales : c'est un mélange subtil d'habitudes et de valeurs ; c'est aussi le fruit d'une longue maturation, dans laquelle les éléments successifs viennent ajouter à la richesse de l'ensemble. » Jacqueline de Romilly, Le jardin des mots.
Inspirer le désir de découvrir la langue qui a contribué à l'éclat de la littérature grecque antique, tel est le souhait des auteurs de ce livre. Au cours de ces huit leçons autour du grec ancien, le lecteur comprendra mieux aussi pourquoi cette langue est, aujourd'hui encore, à la base de nos classements intellectuels et forme le noyau du vocabulaire scientifique de l'Europe. 


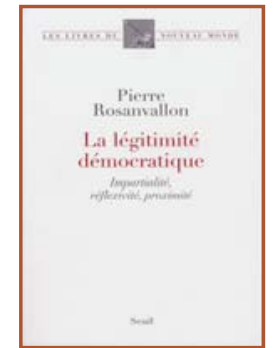

La légitimité démocratique Impartialité, réflexivité, proximité Pierre Rosanvallon

Coll. Les livres du Nouveau monde Paris, Éditions du Seuil, 2008

Le peuple est la source de tout pouvoir démocratique. Mais l'élection ne garantit pas qu'un gouvernement soit au service de l'intérêt général, ni qu'il y reste. Le verdict des urnes ne peut donc être le seul étalon de la légitimité. Les citoyens en ont de plus en plus fortement conscience. Une appréhension élargie de l'idée de volonté générale s'est ainsi imposée. Un pouvoir n'est désormais considéré comme pleinement démocratique que s'il est soumis à des épreuves de contrôle et de validation à la fois concurrentes et complémentaires de l'expression majoritaire. Il doit se plier à un triple

Aristote

Jean-Marie Zemb

Traduit de l'allemand par François et

Robert Muller

Limoges, Éd. Lambert-Lucas, 2008.

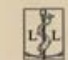

«Ce sont les quatre questions fondamentales qu'Aristote s'est posées - D'où ? Comment? Quoi ? En vue de quoi ? - et dont il a recommandé l'usage, et non le déroulement chronologique de sa vie, qui nous permettront, dans le cadre de cette monographie, de rassembler les témoignages provenant d'Aristote luimême, afin de faire son portrait. La philosophie d'Aristote constitue une unité, parce qu'elle s'est développée de façon organique, parce qu'elle est formellement élaborée, parce qu'elle n'exclut aucun objet possible et parce qu'en elle l'homme poursuit un but. »

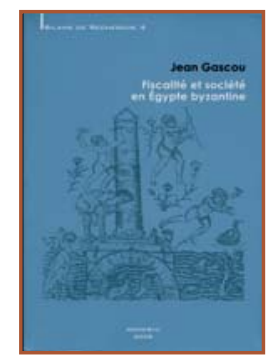

Fiscalité et société en Égypte byzantine Jean Gascou

Coll. Bilans de recherche 4

Ouvrage publié avec le concours de la

Fondation Ebersolt du Collège de

France

Paris, Centre d'histoire et civilisation de Byzance, 2008. impératif de mise à distance des positions partisanes et des intérêts particuliers (légitimité d'impartialité), de prise en compte des expressions plurielles du bien commun (légitimité de réflexivité), et de reconnaissance de toutes les singularités (légitimité de proximité). D'où le développement d'institutions comme les autorités indépendantes et les cours constitutionnelles, ainsi que l'émergence d'un art de gouvernement toujours plus attentif aux individus et aux situations particulières.

Nous sommes entrés dans ce nouveau monde sans en avoir perçu la cohérence ni interrogé lucidement les problèmes et les conséquences. C'est à en dévoiler les ressorts et les implications que s'emploie ici Pierre Rosanvallon. Après avoir publié La Contre-démocratie (Seuil, 2006), il propose, dans ce deuxième volet de son enquête sur les mutations de la

L'ouvrage : Aristoteles in Selbstzeugnissen und Bilddokumenten a été publié à Reinbek (Hambourg) en 1961 par Rowohlt Verlag. Devenu un classique des études de philosophie antique, en Allemagne, il a été constamment réédité depuis. Une édition japonaise est parue en 1967, une édition grecque en 1979 et une édition coréenne en 2004.

L'auteur : Jean-Marie Zemb (1928-2007), a été professeur au Collège de France, titulaire de la chaire de Grammaire et pensée allemandes de 1986 à 1998.

Les traducteurs : François Muller, professeur d'allemand à l'université Paris X - Nanterre, ancien élève de J.-M. Zemb ; Robert Muller, professeur de philosophie à l'université de Nantes, spécialiste de philosophie grecque.

\section{Autres publications}

On trouvera ici vingt-trois études de papyrologie byzantine jalonnant plus de trente ans de vie scientifique, souvent mises à jour, nuancées ou corrigées. Techniques et ponctuelles, elles se fondent néanmoins dans une perspective commune : l'élucidation des structures agraires et de la fiscalité de l'Égypte byzantine. L’auteur serait satisfait si cet ouvrage permettait aux spécialistes de la haute époque byzantine de saisir l'unité de son dessein. 

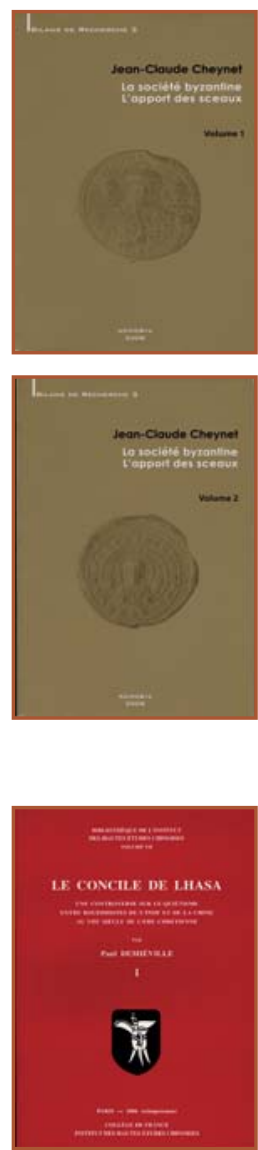

Le Concile de Lhasa. Une controverse sur le quiétisme entre bouddhistes de l'Inde et de la Chine au VIIIe siècle de l'ère chrétienne.

Paul Demiéville

Collège de France, Institut des hautes études chinoises, Bibliothèque de l'Institut des hautes études chinoises, volume VII. 410 p., 32 pl. h.-t., 1952-1987, avec avertissement et corrigenda, réimpression 2006.

L'Institut des hautes études chinoises du Collège de France a imprimé pour la troisième fois l'ouvrage de Paul Demiéville (1894-1979): Le Concile de Lhasa. Une controverse sur le quiétisme entre bouddhistes de l'Inde et de la Chine comporte une introduction à la sigillographie byzantine destinée à un large public ainsi que des études sur les institutions économiques, sociales ou militaires et sur certains aspects de la piété personnelle. Le second volume regroupe des recherches sur des familles aristocratiques de tradition militaire, dont la plupart ont fourni des empereurs ou leur ont été apparentées. Un index exhaustif des noms en fin de volume, témoin de l'apport de la sigillographie à la prosopographie byzantine, sera un outil commode pour les chercheurs.

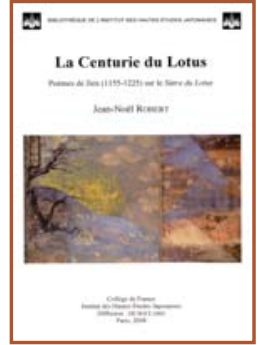

La Centurie du Lotus

Poèmes de Jien (1155-1225) sur le Sûtra du Lotus

Jean-Noël Robert

Bibliothèque de l'Institut des hautes études japonaises

Institut des hautes études japonaises du

Collège de France

Paris, Éditions de Boccard, 2008.

Ce livre présente la traduction et le commentaire de 170 poèmes composés en langue japonaise par le moine Jien (1155-1225), poète et érudit médiéval, sur cent citations de la version chinoise du Sûtra du Lotus. En mettant en œuvre toutes les ressources de la poétique traditionnelle japonaise, Jien donne une série de pièces dont presque chaque mot entre en au VIIIe siècle de l'ère chrétienne. L'ouvrage a été publié pour la première fois en 1952. Après épuisement, il a été réimprimé en 1987 au Collège de France dans la collection "Bibliothèque de l'Institut des hautes études chinoises » (Vol. VII), en y intégrant une centaine de corrections et une page d'errata laissées par P. Demiéville dans son exemplaire personnel. Basé pour l'essentiel sur des manuscrits chinois rapportés de Dunhuang par Paul Pelliot, enrichi d'une annotation aussi encyclopédique que passionnante, Le Concile de Lhasa est depuis longtemps un « classique » de la sinologie française, tant pour les bouddhologues sinisants ou sanskritistes (P. Demiéville était l'un et l'autre) que pour les tibétologues. Cette réimpression est identique à celle de 1987. résonance avec le texte chinois d'une part, mais aussi avec la poésie japonaise antérieure, tout en fournissant, sous le couvert de l'imagerie littéraire, des interprétations doctrinales fondamentales de la lettre du sûtra.

Le commentaire vise à éclaircir les principales correspondances sémantiques et dogmatiques de ces "chants japonais" au sens inépuisable. À travers l'étude de cet important corpus de poèmes japonais à thème bouddhique (shakkyôka), c'est tout un aspect méconnu de la relation linguistique et religieuse sino japonaise qui est mis en lumière. 